\title{
Designing Interactions for the Ageing Populations - Addressing Global Challenges
}

\author{
Sayan Sarcar \\ University of Tsukuba, Japan \\ Jussi Jokinen \\ University of Helsinki, Finland
}

\author{
Cosmin Munteanu \\ University of Toronto Mississauga, \\ Canada \\ Xiangshi Ren \\ Kochi University of Technology, \\ Japan
}

\author{
Neil Charness \\ Florida State University, USA \\ Emma Nicol \\ University of Strathclyde, UK
}

\begin{abstract}
We are concurrently witnessing two significant shifts: digital devices are becoming ubiquitous, and older people are becoming a very large demographic group. However, despite the recent increase in related $\mathrm{CHI}$ publications, older adults continue to be underrepresented in HCI research as well as commercially. Therefore, the overarching aim of this workshop is to increase the momentum for such research within $\mathrm{CHI}$ and related fields such as gerontechnology. For this, we plan to continue developing a space to discuss and share principles and strategies to design interactions and evaluate user interfaces (UI) for the ageing population. We thus welcome contributions of proposing improved empirical studies, theories, design and evaluation of UIs for older adults. Building on the success of the last three years' workshops, we aim to grow the community of $\mathrm{CHI}$ researchers across borders interested in this topic by fostering a space to exchange results, methods, approaches, and ideas from research on interactive applications in support of older adults that are reflective of international diversity.
\end{abstract}

\section{CCS CONCEPTS}

- Human-centered computing; • Interaction design; • Interaction design process and methods;

\section{ACM Reference Format:}

Sayan Sarcar, Cosmin Munteanu, Neil Charness, Jussi Jokinen, Xiangshi Ren, and Emma Nicol. 2021. Designing Interactions for the Ageing Populations - Addressing Global Challenges. In CHI Conference on Human Factors in Computing Systems Extended Abstracts (CHI '21 Extended Abstracts), May 08-13, 2021, Yokohama, Japan. ACM, New York, NY, USA, 4 pages. https: //doi.org/10.1145/3411763.3441326

\section{INTRODUCTION}

Both developed and developing nations are facing rapid ageing of their populations: people aged over 65 years old are expected to comprise $27 \%$ and $17 \%$ of these nations' population, respectively, by $2050[3,5]$. Although issues related to older adults are receiving substantial attention in other areas of research e.g., Gerontology,

Permission to make digital or hard copies of part or all of this work for personal or classroom use is granted without fee provided that copies are not made or distributed for profit or commercial advantage and that copies bear this notice and the full citation on the first page. Copyrights for third-party components of this work must be honored For all other uses, contact the owner/author(s).

CHI '21 Extended Abstracts, May 08-13, 2021, Yokohama, Japan

(C) 2021 Copyright held by the owner/author(s)

ACM ISBN 978-1-4503-8095-9/21/05.

https://doi.org/10.1145/3411763.3441326
Accessibility, Cognitive Psychology, the HCI community might contribute more. Ageing is associated with a multitude of biological, cognitive, and social changes that impact the use of technology [4]. Age also brings new opportunities, which well-designed UIs could support, such as increased spare time, strengthened family connections, and new learning and travel opportunities. However, the digital revolution has not adequately considered the needs of ageing populations. Two trends have been observed now, which threaten adoption, use, and engagement with technology by older adults: (i) size of digital devices, over time has been decreasing (from Desktop to Tablet, Smartphone to Smartwatch) and (ii) after the IoT revolution, small, sensor-based devices have become ubiquitous. Due to normative decline in sensorimotor abilities with age, both trends disadvantage older adults' access to those devices. Although older adults constitute an increasing demographic segment, the majority of research in HCI, as well as by technology companies, focuses almost exclusively on younger adults. As a result, even though digital device ownership among older adults has significantly increased in recent years [2], device adoption level is still low (for example., smartphone ownership in the US is at $53 \%$ for older adults as of 2019 , whereas $96 \%$ of young people aged 18-29 are smartphone owners [2]). Consequently, older adults may be losing the possible benefits and opportunities from this growing digital era $[1,4,6]$.

This submission builds on successful workshops at CHI 2018 and CHI $2020[16,18]$. We aim to enrich our collective knowledge and body of practice by fostering an inter-disciplinary and international space for idea sharing and community building. New for 2021, we strive to include HCI researchers whose work is applicable to older adults and is representative of geographic and cultural diversity within $\mathrm{CHI}$ and, specifically, the socio-economic and cultural contexts surrounding broad categories of older adults. For example, this may include those working on interactive AI systems or voice assistants, or those studying empowering technologies within the Global South. The goal of this workshop is to discuss suitable design and evaluation methodologies of UIs, fostering engagement with digital devices as well as coordinating efforts to raise awareness of $\mathrm{HCI}$ as it affects aging adults, and to rethink designing and evaluating diverse senior-centred interfaces.

\section{FOCUS AREAS}

This workshop focuses on discussing different UI design methodologies (as in ways of investigating design/use), which will help older adults to access current digital technologies. For example, the 
"mobile device" is one of the prominent current and future forms of mobile computing, including phones, tablets, and wearables. Mobile device ownership rates for older adults are increasing [2], yet there are fewer concrete principles for designing for older adults $[1,4]$. Voice assistants (e.g. Alexa) are advertised as helpful for seniors, yet they are not designed for seniors. Current UI designs are required to be culturally and personally relevant to specific groups. For example, one of the relevant problems is in the "OOBE" (Out Of Box Experience), setting up the device in the first place. There are few instructions, and they are generally not step-by-step. They leave out details, or refer older adults to a website meaning they have to coordinate between the computer they use to access the website and the devices they are using for setup: smartphone, smart speaker). Older adults need to be adept with a smartphone device (download the app, set it up), then able to link the device to their home network (if they have one). This is way too complicated a procedure for a novice older user to solve (as evidenced by a Pew survey on the degree to which different age groups agreed or strongly agreed with statements about having difficulty setting up new devices [19]). Also, cultural issues pale in comparison. In addition, there are the management/maintenance issues when a power failure occurs, and you need to bring up your network and your linked devices, and management also from the perspective of cybersecurity and the need to update firmware/bios on IoT devices. Few people consider the entire infrastructure surrounding a specific device's deployment.

Further, evaluating both the usability and the social and personal benefits of older adult friendly mobile and other intelligent interfaces (e.g., conversational UI, IoT interface, AI systems) is challenging and not well supported by existing HCI [6]. Overall, we think that better access to information through digital devices will provide older people with more opportunities for social connection and an enhanced sense of belonging in digital society.

\section{WORKSHOP GOALS}

$\mathrm{ACM} \mathrm{CHI}$ is one of the most multidisciplinary research communities. In recent years it has made tremendous progress in supporting many marginalized user groups (e.g. low literacy, developing countries, accessibility) or in addressing critical societal needs (e.g. sustainability, inclusion). It is now timely to more systemically investigate how we, as a community, can leverage such advances in research and design and better support the safety, health, social, or digital inclusion needs of older adults. While recent years have seen an increase in such research activity at CHI, it has mostly materialized in the form of isolated publications. Yet there is increasinglystrong evidence that $\mathrm{HCI}$ researchers are becoming interested in this topic. This is exemplified by the very large attendance (more than 50 participants) to our first Special Interest Group (SIG) on Older Adults, held at CHI 2016 [13], or by the numerous submissions received by recent workshops such as Re-imagining commonly used mobile interfaces for older adults in MobileHCI 2014 [11], and 2nd Workshop on Designing with Older Adults: Towards a Complete Methodology in MobileHCI 2015 [12] or the first-ever international symposium on Interactive Technology and Ageing Populations [14], held in October 2016. As a continuation, we conducted a workshop on Designing Mobile Interactions for the Ageing Populations at
CHI 2017 [15] followed by the workshop on Designing Interactions for the Ageing Populations at CHI 2018 [16], which attracted people from diversified areas like Gerontology, Accessibility, and Psychology. We organized a recent follow-up workshop at $\mathrm{CHI}$ 2020 [18] which includes discussions on design and engagement issues of current senior-centred UIs specific to the diverse abilities of older people at different ages (difference in digital device usage between $65+, 75+$ and $85+$ years of age) and cultural diversities. All these events were co-organized by the proponents of this proposal. Capitalizing on this recent increase in interest, this workshop aims to reach three goals:

Enriching the Research Repository

In our CHI 2018 and CHI 2020 workshops, researchers from various fields participated in synthesizing and collating findings from different disciplines, and discussed efficient, effective, usable, and adoptive technologies and more appropriate methods. In this workshop, we will enrich the repository by providing further sights about the implications of interface design in digital devices.

Continuing Community Building

Senior-centred research and development is currently conducted in academic and industry research labs in a rather disjointed manner. In common with the last three years, this workshop's goal is also to link the SIGCHI community with researchers and practitioners across academic disciplines (such as the Gerontology, Cognitive Science, Psychology, Sociology, Cognitive Neuroscience, Culturebased Design) and industries who are actively working or have interest toward understanding older adults' technology use. For future collaborations, mailing lists and post-CHI activities (e.g., a symposia/summer school) will be established.

Raising Awareness

Interactive technologies (e.g., Amazon Alexa, IoT systems) for seniors is a significant market of interest for industries, expected to grow from US\$ 2 billion to an estimated US\$ 30 billion in the next few years [7]. This is a natural reflection of the size of this user group (e.g., $17.5 \%$ of the population are of $65+$ years of age in Canada [8]). Yet interest in HCI is still relatively small (less than $1 \%$ of all CHI 2015 accepted submissions across all tracks can be categorized as focused on older adults). This workshop aims to raise awareness of the challenges and research opportunities in this field, with a renewed focus on aging adults across the globe in diverse socio-economic and cultural contexts.

\section{WORKSHOP THEMES}

We suggest several relevant themes for guiding participants' papers and discussions during the event.

1. Current Issues

Older adults face many challenges while accessing UI in digital devices. This topic will focus on discussing issues related to human factors, perception, memory, and motor movement of older adults, and how these issues affect the design of senior-based UIs.

2. Opportunities

Various models of technology acceptance suggest that adoption is facilitated if users, especially older adults, see value in starting to use that technology. As such, we will initiate discussions on identifying opportunities to sustain some of the current activities that 
older adults engage in, and on determining how to better support these activities through appropriately-designed interactions or UIs.

3. Social benefits

Many seniors live increasingly isolated lives, not only physically, but also without a strong social network. This is expected to become increasingly critical as the adoption rate of new technologies that could support social connectivity [18] decreases with age (especially in retirement). We will discuss design opportunities and challenges to facilitating social connectivity and social participation (e.g. in family life) by older adults.

4. Models and Design

Various theories (perceptual, cognition, motor movement) and design principles (e.g., participatory design, ability-based design [10, 17]) have been proposed to develop UIs for older adults. This topic will focus on discussing the existing models and design principles for UIs of older adults. For example, as current mobile interfaces tend to follow a "one design for all" approach, model parameters can be further tuned to cover individual differences among aging adults and ability-based design and optimization principles can be used to find effective UI design for this group.

5. Evaluation Methodologies

Evaluating senior-based UIs still faces many challenges, particularly in accurately understanding the preferences, habits, and adoption challenges of older adults with digital devices [4]. For example, there is growing evidence that younger adults help their grandfather or grandmother to learn, and encourage their use of technology [9]. More accurate questionnaire and ethnographic studies are required in order to understand people's behavior to interact with different digital devices more effectively (e.g., using remote switch to turn on digital appliances), and to understand the challenges in developing countries, such as older adults facing issues dealing with cashless transactions on mobile devices. This topic will focus on discussing existing evaluation methods, to explore how suitable current methods are for judging the efficacy of UI design, and will identify future research scopes.

5. Applications

Digital devices open up many new possibilities and opportunities for older adults. This topic will discuss what some potentially useful applications for older adults are. For examples, text-entry methods on mobile devices can enhance the usability of messaging applications. Games and social VR applications have the potential to improve the wellbeing of older adults. We will conclude with a list of future opportunities for apps.

6. Cross-Cultural Aspect

A cross-cultural perspective of Healthy Ageing becomes important in the current senior-centred research agenda as elderly people living in different parts of the world possess both common and unique perspectives regarding technology usage and adoption. We will look at culture effects in the co-design and participatory design methods of applications.

We welcome all HCI topics related to older adults and technologies. The topics include, but are not limited to:

- Effects of decline in perceptual, cognitive, and motor performance on digital device use

- Models of user performance
- Understanding device ecology and leveraging it to design suitable interfaces

- UI design patterns and metaphors

- Opportunities to sustain older adults' current activities and practices through digital technologies

- Societal implications, including digital divide and social exclusion

- Bridging Social Computing and senior-centred HCI4D

- Evaluation methodologies

- Ethical concerns

- Senior engagement in Design

- Diversity in Ageing

Several key questions will be discussed in the workshop, e.g., 1) How does user behavior for performing tasks with several digital devices (e.g., mobile device, TV remote, home appliances) change across age? 2) How well can psychological theory capture individual differences in older adult task behavior? 3) What is the basis for proposing application design guidelines for older adults in digital devices? 4) Can mathematical and simulation models automatically design a UI adaptive to older user behavior? (5) what are the possible methodological approaches for a meaningful engagement of older adults in design, (6) how to design by considering diversity aspects in aging. At the workshop, we intend to showcase some topicrelated research projects through short presentations of the authors as well as plenary discussions and birds of a feather groups, focusing on areas of interest that are important to the wider older adult community.

\section{REFERENCES}

[1] Farah Arab, Yasir Malik, and Bessam Abdulrazak. 2013. Evaluation of phonage: an adapted smartphone interface for elderly. In Proceedings of $18^{\text {th }}$ International IFIP TC-13 Conference on Human-Computer Interaction (INTERACT 2013), September 2-6, Cape Town, South Africa, Springer, Heidelberg, Germany, 547-554. DOI: http://dx.doi.org/10.1007/978-3-642-40498-6 44

[2] Mobile fact sheet. 2021. Pew Research Center. Retrieved from https://www. pewinternet.org/fact-sheet/mobile/.

[3] Miranda A Farage, Kenneth W Miller, Funmi Ajayi, and Deborah Hutchins. Design principles to accommodate older adults. Global journal of health science. 4, 2 (March 2012). DOI: http://dx.doi.org/10.5539/gjhs.v4n2p2

[4] Sara J Czaja, Walter R. Boot, Neil Charness,and Wendy A Rogers. 2019. Designing for older adults: Principles and creative human factors approaches (3rd Edition). CRC press, FL, USA.

[5] National Institute of Health. National Institute of Health Report. 2020. Retrieved from https://www.nih.gov/news-events/news-releases/worlds-older-populationgrows-dramatically/

[6] Barbara B. Neves, Rachel L. Franz, Cosmin Munteanu, Ronald Baecker, and Mags Ngo. 2015. "My hand doesn't listen to me!": adoption and evaluation of a communication technology for the 'oldest old'. In Proceedings of the ACM SIGCHI Conference on Human Factors in Computing Systems (CHI 2015), Seoul, South Korea, ACM Inc., New York, NY, 1593-1602. DOI: http://doi.acm.org/10. 1145/2702123.2702430

[7] Aging in Place Technology Watch. 2020. Retrieved from https://www. ageinplacetech.com/files/aip/Market

[8] Stats Canada Population Estimates, 2020. Retrieved from https://www150.statcan. gc.ca/n1/daily-quotidien/190930/dq190930a-eng.htm.

[9] Marilyn R. McGee-Lennon, Maria K Wolters, and Stephen Brewster. 2011. Usercentred multimodal reminders for assistive living. In Proceedings of the ACM SIGCHI Conference on Human Factors in Computing Systems (CHI 2011), Vancouver, Canada, ACM Inc., New York, NY, 2105-2114. DOI: http://doi.acm.org/10. 1145/1978942.1979248

[10] Sayan Sarcar, Jussi Joklnen, Antti Oulasvirta, Chaklam Silpasuwanchai, Zhenxin Wang, and Xiangshi Ren. 2016. Towards Ability-Based Optimization for Aging Users. In Proceedings of the International Conference on Interactive Technology and Aging Populations (ITAP '16), Kochi, Japan, ACM Inc., New York, NY, 77- 86. DOI: http://dx.doi.org/10.1145/2996267.2996275 
[11] Emma Nicol, Mark D. Dunlop, Andreas Komninos, Marilyn Rose McGee-Lennon, Lynne Baillie, Alistair Edwards, Parisa Eslambolchilar, Joy Goodman-Deane, Lilit Hakobyan, Jo Lumsden, Ingrid Mulder, Patrick Rau, Katie A. Siek. Re-imagining commonly used mobile interfaces for older adults. In Proceedings of ACM SIGCHI Conference on Mobile Devices \& Services (MobileHCI '14), Toronto, Canada, ACM Inc., New York, NY, 585-588. DOI: http://dx.doi.org/10.1145/2628363.2634261

[12] Emma Nicol, Mark D. Dunlop, Andreas Komninos, Marilyn Rose McGee-Lennon, Lynne Baillie, Parisa Eslambolchilar, Pin Sym Foong, Paul Gault, Lilit Hakobyan, Jo Lumsden, Fáber Danilo Giraldo Velásquez, Ann-Marie Horcher. Designing with Older Adults: Towards a Complete Methodology. In Proceedings of ACM SIGCHI Conference on Mobile Devices \& Services (MobileHCI '15). ACM Inc. New York, NY, 908- 911. DOI: http://dx.doi.org/10.1145/2786567.2795395

[13] Neil Charness, Mark D. Dunlop, Cosmin Munteanu, Emma Nicol, Antti Oulasvirta Xiangshi Ren, Sayan Sarcar, Chaklam Silpasuwanchai, In Proceedings of Extended Abstracts of the ACM SIGCHI Conference on Human Factors in Computing Systems (CHI 2016), San Jose, USA, ACM Inc., New York, NY, 1131-1134. DOI: http://dx.doi.org/10.1145/2851581.2886431

[14] International Symposium on Interactive Technology and Ageing Populations (IxAP 2016). 2016. Retrieved from http://itap2016.xrenlab.com/.

[15] Sayan Sarcar, Cosmin Munteanu, Jussi P. P. Jokinen, Antti Oulasvirta, Chaklam Silpasuwanchai, Neil Charness, Mark D. Dunlop, Xiangshi Ren. , In Proceedings of Extended Abstracts of the ACM SIGCHI Conference on Human Factors in Computing Systems (CHI 2017), Denver, USA, ACM Inc., New York, NY, 11311134. DOI: http://dx.doi.org/10.1145/3027063.3027074

[16] Sayan Sarcar, Cosmin Munteanu, Jussi Jokinen, Antti Oulasvirta, Neil Charness, Mark D. Dunlop, Xiangshi Ren, In Proceedings of Extended Abstracts of the ACM SIGCHI Conference on Human Factors in Computing Systems (CHI 2018), Montreal, Canada, ACM Inc., New York, NY, W24, DOI: http://dx.doi.org/10.1145/ 3170427.3170607

[17] Sayan Sarcar, Jussi Joklnen, Antti Oulasvirta, Zhenxin Wang, Chaklam Silpasuwanchai, and Xiangshi Ren. 2018. Ability-based Optimizations for Touchscreen Interactions, IEEE Pervasive Computing Magazine, vol. 17, 1 (January 2018), pp. 15-26.

[18] Sayan Sarcar, Cosmin Munteanu, Jussi Jokinen, Neil Charness, Mark D. Dunlop, Xiangshi Ren, Jenny Waycott. In Proceedings of Extended Abstracts of the ACM SIGCHI Conference on Human Factors in Computing Systems (CHI 2020), Honolulu, USA, ACM Inc., New York, NY, W21, DOI: https://dl.acm.org/doi/abs/10. $1145 / 3334480.3375164$

[19] Tech Adoption Climbs Among Older Adults. 2017. Retrieved from http://www. pewinternet.org/2017/05/17/tech-adoption-climbs-among-older-adults/ 